\title{
Amyotrophic Lateral Sclerosis with an Acute Hypertensive Crises
}

\author{
Ha Lim Lee, M.D., Ju Kang Lee, M.D., Ph.D. \\ Department of Rehabilitation Medicine, Gachon University of Medicine and Science, Incheon 405-760, Korea
}

Amyotrophic lateral sclerosis (ALS) is a progressive neurodegenerative disorder involving the systemic motor neurons, but autonomic nervous function is relatively well preserved. A few studies related to autonomic dysfunction have been reported, but autonomic dysfunction is rare in ALS. Moreover, dysautonomia symptoms are not prominent in patients with ALS. We present a 55-year-old male patient with ALS, who had acute severe hypertension and tachycardia crises, as well as sudden falls in his blood pressure. After he was diagnosed with ALS, he suddenly collapsed and was placed under mechanical ventilation. Several hypertensive attacks and dysautonomic signs then occurred. We successfully controlled the dysautonomia using diazepam and doxazocin mesylate, an alpha receptor antagonist.

Key Words Amyotrophic lateral sclerosis, Autonomic dysfunction, Hypertensive attack

\section{INTRODUCTION}

Amyotrophic lateral sclerosis (ALS) is a progressive neurodegenerative disorder involving the systemic motor neurons, but the autonomic nervous function is relatively well preserved. ${ }^{1}$ However, symptoms of dysautonomia have been reported in patients with ALS and evidence of autonomic changes has been disclosed. ${ }^{1,2} \mathrm{~A}$ number of studies have shown impaired cardiac autonomic control in patients with ALS together with parasympathetic dysfunction and sympathetic predominance. ${ }^{3}$ Disturbances

Received December 22, 2010; Accepted August 22, 2011

Corresponding author: Ju Kang Lee

Department of Rehabilitation Medicine, Gachon University of Medicine and Science, 1198, Guwol-dong, Namdong-gu, Incheon 405-760, Korea

Tel: +82-32-460-3722, Fax: +82-32-460-3722, E-mail: pm@gilhospital.com

(c) This is an open-access article distributed under the terms of the Creative Commons Attribution Non-Commercial License (http:// creativecommons.org/licenses/by-nc/3.0) which permits unrestricted noncommercial use, distribution, and reproduction in any medium, provided the original work is properly cited.

Copyright ( 2012 by Korean Academy of Rehabilitation Medicine in autonomic cardiac control in respirator-dependent patients with ALS may significantly influence survival and may lead to a hypertensive crisis, circulatory collapse, and sudden death. ${ }^{4}$ We present here a rare case of a patient with ALS and hypoxic brain injury after cardiac arrest who developed marked blood pressure (BP) and heart rate (HR) fluctuations, which were controlled by daily administration of diazepam and doxazocin mesylate.

\section{CASE REPORT}

A 55-year-old man was admitted to our hospital complaining of progressive weakness of the upper extremities, dysarthria, and dysphagia for 6 months. He had a history of hypertension, for which he had intermittently taken antihypertensive medicine. He showed limb muscle atrophy, fasciculation of the tongue, and weakness in all extremities. The proximal muscles of both upper extremities showed a Medical Research Council (MRC) 
grade of 2 and the distal muscles showed a MRC grade of 3 . The muscles of both lower extremities had a MRC grade of 4. Deep tendon reflexes were hyporeflexive in both upper limbs and hyper-reflexive in both lower limbs. No pathological reflexes were observed, and sensory function was normal. Results of a nerve conduction

Table 1. Results of Nerve Conduction Studies

\begin{tabular}{|c|c|c|c|c|}
\hline & Stimulation & $\begin{array}{c}\text { Latency } \\
\text { (ms) }\end{array}$ & $\begin{array}{c}\text { Amplitude } \\
(\boldsymbol{\mu V})\end{array}$ & $\begin{array}{c}\text { Velocity } \\
(\mathrm{m} / \mathrm{s})\end{array}$ \\
\hline \multicolumn{5}{|l|}{ Motor nerve } \\
\hline \multirow[t]{2}{*}{ Rt median } & Wrist & 3.80 & 0.80 & \\
\hline & Elbow & 7.55 & 0.70 & 56.0 \\
\hline \multirow[t]{2}{*}{ Rt ulnar } & Wrist & 3.95 & 3.50 & \\
\hline & Elbow & 7.35 & 3.40 & 67.6 \\
\hline \multirow{2}{*}{$\begin{array}{l}\text { Lt peroneal } \\
\text { (EDB) }\end{array}$} & Ankle & 3.30 & 11.60 & 52.4 \\
\hline & FH & 5.40 & 7.10 & \\
\hline \multirow{2}{*}{$\begin{array}{l}\text { Lt tibial } \\
(\mathrm{AH})\end{array}$} & Ankle & 4.70 & 5.50 & 67.6 \\
\hline & $\mathrm{PF}$ & 11.95 & 5.50 & \\
\hline \multicolumn{5}{|l|}{ Sensory nerve } \\
\hline Rt median & Wrist & 3.40 & 24.0 & 56.0 \\
\hline Rt ulnar & Wrist & 3.05 & 22.0 & 57.1 \\
\hline Rt radial & Forearm & 1.95 & 61.0 & 62.1 \\
\hline Lt sural & Ankle & 2.45 & 16.1 & 64.5 \\
\hline $\begin{array}{l}\text { Lt superficial } \\
\text { peroneal }\end{array}$ & Ankle & 2.45 & 23.2 & 58.1 \\
\hline
\end{tabular}

EDB: Extensor digitorum brevis, AH: Abductor hallucis, FH: Fibular head, PF: Popliteal fossa study and needle electromyography were consistent with motor neuron disease (Tables 1, 2). Based on his clinical features and the results of electrodiagnostic tests, he was diagnosed with ALS. He could not sleep well because of hypercapnia, which was noted on a blood gas analysis $\left(\mathrm{PaCO}_{2}, 52.1 \mathrm{mmHg}\right)$. Dysphagia was confirmed by a video-fluoroscopic swallow study. His diurnal BP and HR variations were stable. On day 15 of hospitalization, he suddenly collapsed during an attempt to insert a nasogastric tube. After cardiopulmonary resuscitation (CPR), he was eventually placed under mechanical ventilation with a tracheostomy in the intensive care unit. His mental status was comatose. Magnetic resonance imaging of the brain showed diffuse hypoxic brain damage (Fig. 1).

On day 19 of hospitalization, he suddenly developed a hypertensive attack at 11:30 pm. BP increased to $191 / 108 \mathrm{mmHg}$, HR was $145 / \mathrm{min}$, and respiratory rate (RR) reached $40 / \mathrm{min}$. We used intravenous nicardipine, which is a calcium channel blocker, to lower the BP. But subsequently, BP dropped by $52 / 34 \mathrm{mmHg}$ after 1 hour. BP returned to the normal range several hours later after administration of a saline and dopamine drip infusion (Fig. 2). An ECG revealed sinus tachycardia during the attack. Cardiac markers such as troponin I and creatine kinase-MB levels were normal. He was sweating, facially flushed, shivering, and his pupils were dilated with shallow self respiration efforts. No change was observed on a neurological examination, and the body temperature (BT) was $37.2^{\circ} \mathrm{C}$. On hospitalization day 20, BP increased rapidly again up to $223 / 129 \mathrm{mmHg}$ at $8: 30$ pm. HR increased to $145 / \mathrm{min}$ and RR reached $40 / \mathrm{min}$

Table 2. Results of Needle Electromyography

\begin{tabular}{|c|c|c|c|c|c|c|}
\hline \multirow{2}{*}{ Muscle } & \multicolumn{4}{|c|}{ Abnormal spontaneous activity } & \multirow{2}{*}{ MUAP } & \multirow{2}{*}{$\begin{array}{c}\text { Interferential } \\
\text { patterns }\end{array}$} \\
\hline & IA & Fib & PSW & Fasc & & \\
\hline Rt APB, ADM, FCU & Increased & $2+$ & $2+$ & None & Long/poly & Normal \\
\hline Rt FCR, Biceps, Triceps, Deltoid & Increased & $2+$ & $2+$ & None & Large/long/poly & Normal \\
\hline Rt cervical paraspinalis & Increased & $1+$ & $1+$ & None & - & - \\
\hline Lt TA, PL, GCM (med), VM, TFL & Normal & None & None & None & Normal & Normal \\
\hline Lt lumbar paraspinalis & Normal & None & None & None & - & - \\
\hline Both Tongue & Normal & $1+$ & None & $1+$ & Normal & Normal \\
\hline
\end{tabular}

IA: Insertional activity, Fib: Fibrillation, PSW: Positive sharp wave, Fasc: Fasciculation, MUAP: Motor unit action potential, APB: Abductor pollicis brevis, ADM: Abductor digit minimi, FCU: Flexor carpi ulnaris, FCR: Flexor carpi radialis, TA: Tibialis anterior, PL: Peroneus longus, GCM (med): Gastrocnemius medialis, VM: Vastus medialis, TFL: Tensor fascia lata, Long/poly: Long duration and polyphasic, Large/long/poly: Large amplitude, long duration and polyphasic 

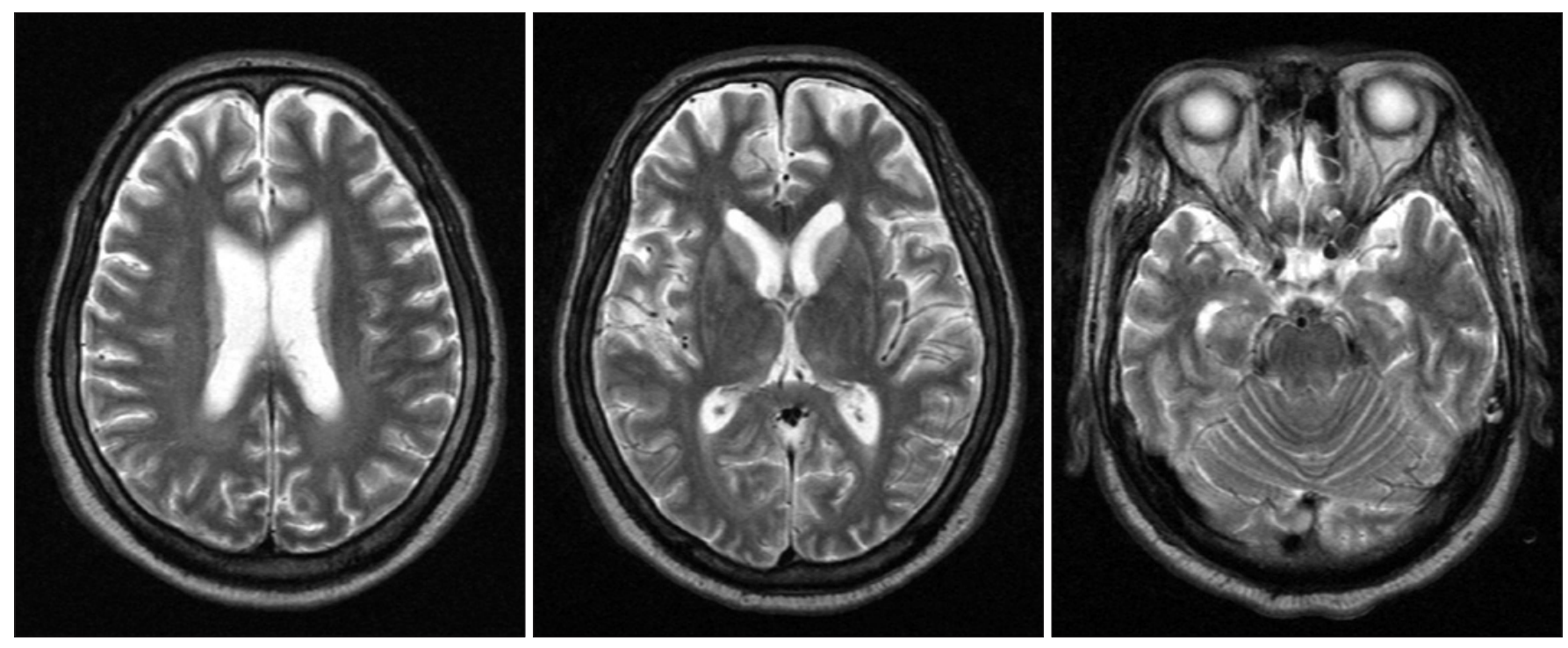

Fig. 1. Brain magnetic resonance imaging shows diffuse hypoxic brain damage.
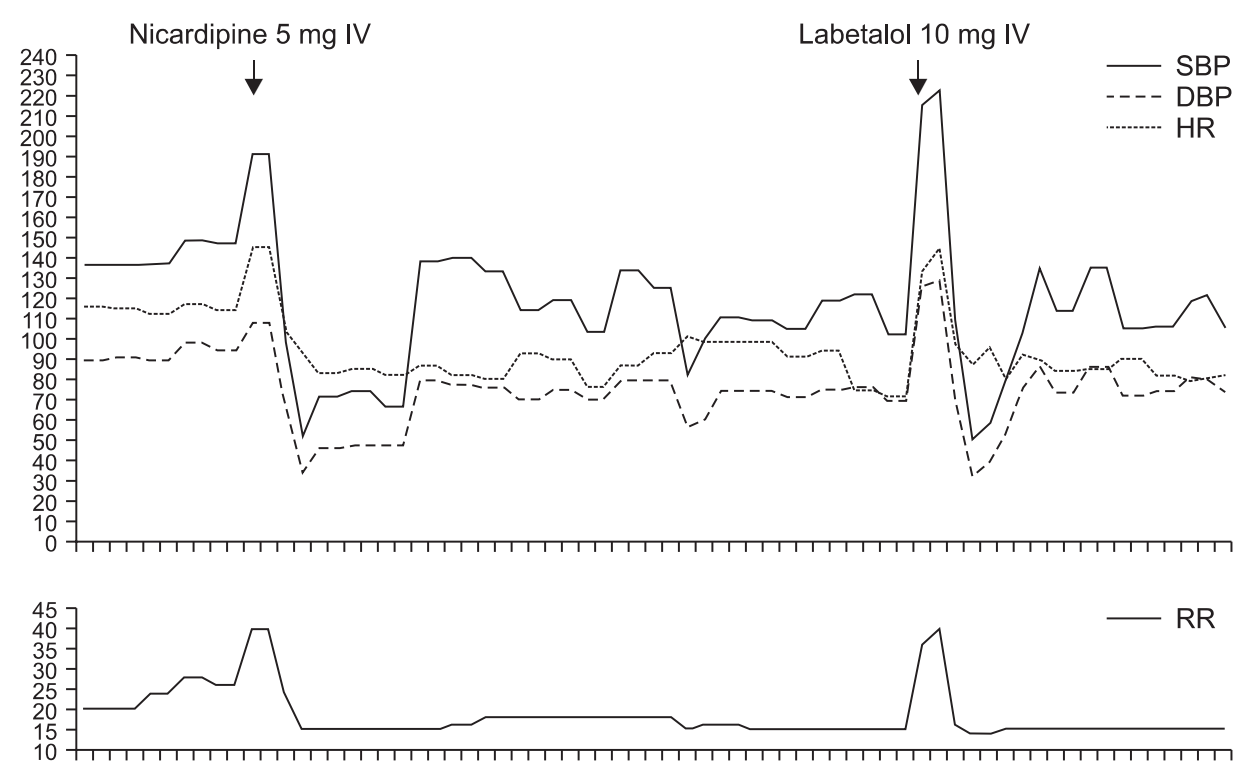

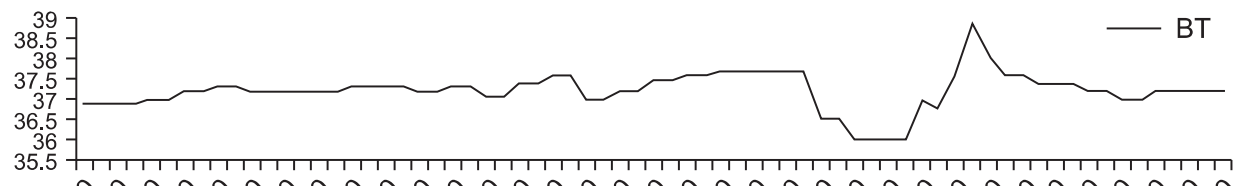

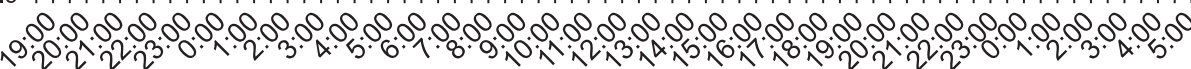

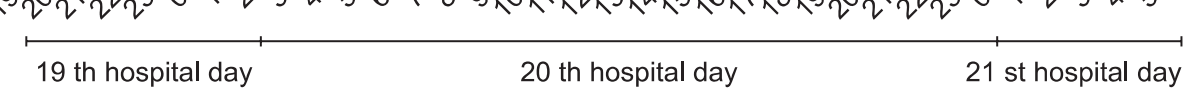

Fig. 2. Alterations in the patient's systolic (SBP), diastolic blood pressure (DBP), heart rate (HR), and body temperature (BT) during hypertensive attacks and the response to intravenous administration of nicardipine (5 $\mathrm{mg})$ and labetalol (10 $\mathrm{mg})$. without a fever $\left(37.0^{\circ} \mathrm{C}\right)$. He also showed sweating, facial flushing, shivering, dilatation of pupils, and shallow self respiration efforts. During the second attack, we used a beta-blocker (10 mg labetalol). The BP dropped again to $50 / 32 \mathrm{mmHg}$ after 1 hour, but the fever rose to $38.9^{\circ} \mathrm{C}$ (Fig. 2). However, no evidence of infection was observed. Based on the clinical course of the two episodes, we thought these hypertensive attacks were related to autonomic dysfunction. We administered 2 $\mathrm{mg}$ /day diazepam, which was more effective to control 


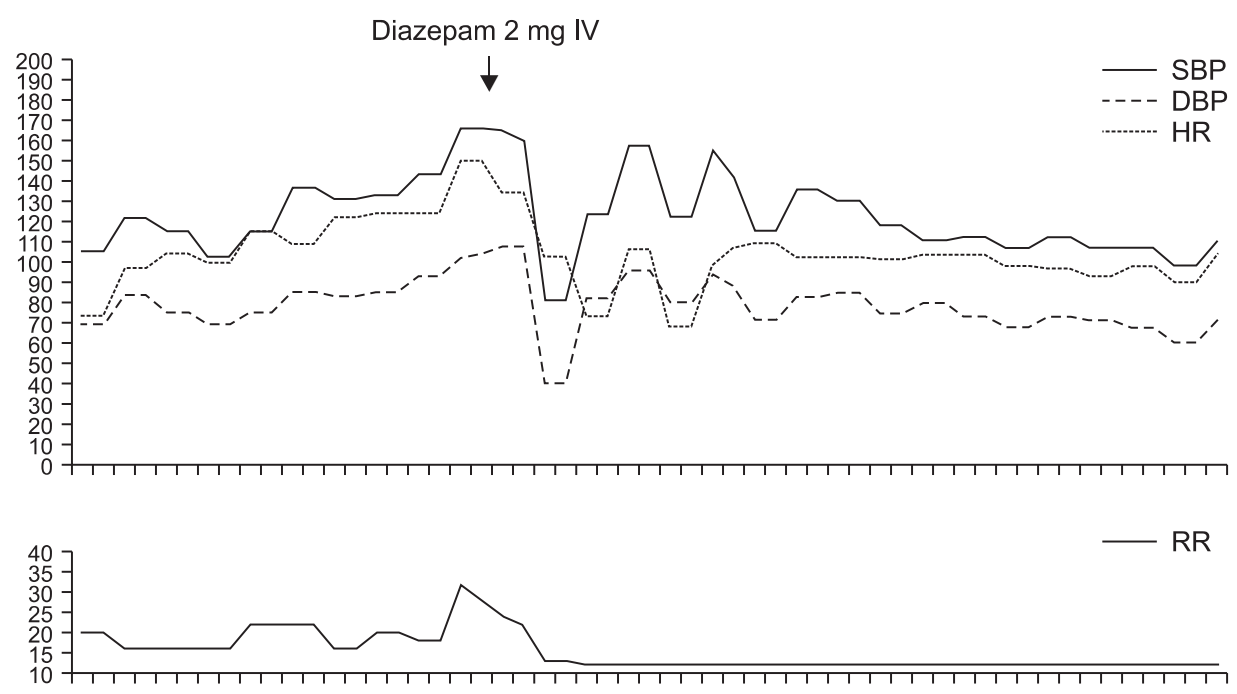

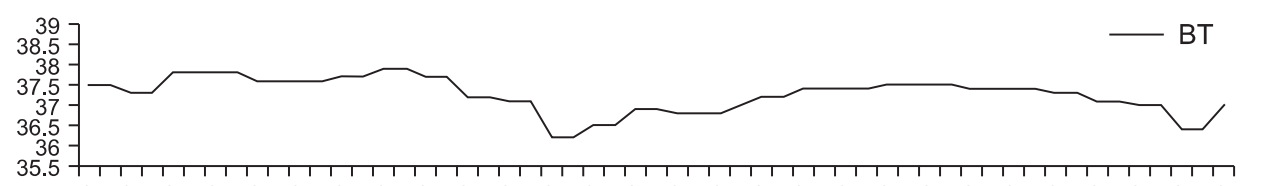

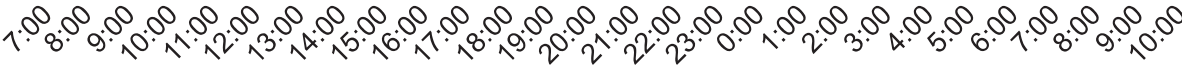

22 th hospital day
Fig. 3. Alterations in the patient's systolic (SBP), diastolic blood pressure (DBP), heart rate (HR), and body temperature (BT) during hypertensive attacks and the response to intravenous administration of diazepam (2 mg). the BP fluctuation than the calcium channel blocker and beta-blocker (Fig. 3). His clinical symptoms improved significantly. We administered $2 \mathrm{mg}$ doxazocin mesylate, which is an alpha receptor antagonist, to prevent another episode of attack. The doses of diazepam and doxazocin mesylate were gradually increased to $6 \mathrm{mg}$ and $4 \mathrm{mg}$, respectively. In the subsequent 2 months he showed several more hypertensive attacks, yet the severity of the attacks progressively lessened on each occasion. We checked the serum level of noradrenaline (NE) at the time of the BP fluctuations and it was within the normal range at $270.31 \mathrm{pg} / \mathrm{ml}$ (normal range, 100-410 $\mathrm{pg} / \mathrm{ml}$ ). The patient's mental status was continuously comatose, and we could not obtain objective evidence of dysautonomia. Although the elevated resting HR and BP continued, the BP eventually repressed.

\section{DISCUSSION}

Symptoms of dysautonomia are not prominent in patients with ALS but have been recently reported in several studies. ${ }^{1-4}$ In previous studies, some advanced and respiratory dependent ALS cases exhibited BP fluctuations, including paroxysmal hypertensive epi- sodes and sudden falls in $\mathrm{BP} .^{2,4}$ Compared with previous studies, more severe dysautonomia symptoms developed in our case. The hypotension observed after intravenous administration of nicardipine and labetalol was marked and more rapid. The BP dropped from $>200 / 120 \mathrm{mmHg}$ to approximately $50 / 30 \mathrm{mmHg}$ within 1 hour. Diurnal $\mathrm{BP}$ variation, daytime hypertension, and night-time hypotension were not seen. The reason for the initial cardiac arrest was unclear, but we considered that dysautonomia might be the primary pathological cause, because he excessively struggled to avoid having a nasogastric tube placed.

Regardless of the reason, hypoxic brain injury occurred in this patient after CPR, and we thought that this might be one of the factors for the marked BP fluctuation. Paroxysmal autonomic instability with dystonia (PAID) can occur after an acquired brain injury and particularly a traumatic or anoxic brain injury. ${ }^{5}$ The clinical features of PAID were quite similar to the dysautonomia in this patient. So, it was difficult to distinguish the main factor causing the severe autonomic dysfunction in this patient. Blackman et al. proposed specific PAID signs such as severe brain injury (Rancho Los Amigos level $\leq \mathrm{IV}$ ), a body temperature of at least $38.5^{\circ} \mathrm{C}, \mathrm{HR}$ of at least 130 beats/ 
min, RR of at least 40 breaths/min, agitation, diaphoresis, and dystonia with return of vital signs to normal between episodes. ${ }^{5}$ However, excitation of the sympathetic system is the major pathophysiology in patients with PAID, and the marked hypotension after hypertension in this case could not be explained. Thus, we conclude by stating that autonomic dysfunction in patients with ALS is a major pathological phenomena and the hypoxic brain damage in our patient might have had an influence as an aggravating factor.

Dysregulation in the limbic system, and particularly the central and basolateral nuclei of the amygdala and hypothalamus, might be responsible for the autonomic dysfunction as the primary pathology in patients with ALS. ${ }^{6}$ Benzodiazepines enhance the inhibitory function of $\gamma$-aminobutyric acid (GABA) in the central nervous system. GABAergic neurons are present in the limbic motor system such as the amygdala and hypothalamus. ${ }^{2}$ Benzodiazepines, including diazepam, show hypotensive action by depressing the central nervous system and this has an effect on baroreflex activity and sympathetic tone. ${ }^{7,8}$ Furthermore, plasma NE concentrations decrease after diazepam administration. ${ }^{8}$ Serum NE level was not elevated during the hypertensive attack in our case. This may have been due to the fact that the NE level was checked after diazepam and doxazocin mesylate were administered. In a previous study, phentolamine (an $\alpha$-adrenergic antagonist) was more effective to reduce $\mathrm{BP}$ than propranolol (a $\beta$-adrenergic antagonist) in a patient with ALS. These results are evidence of an abnormal increase in a-sympathetic tone in patients with ALS, which is supported by increased plasma NE levels. ${ }^{2}$ Therefore, we used diazepam and doxazocine mesylate, and these drugs were effective to repress the BP fluctuations.

We report a case of ALS with dysautonomia and think that physicians must become aware of the presence of severe dysautonomia in patients with ALS.

\section{REFERENCES}

1. Baltadzhieva R, Gurevich T, Korczyn AD. Autonomic impairment in amyotrophic lateral sclerosis. Curr Opin Neuro 2005; 18: 487-493

2. Shimizu T, Kato S, Hayashi M, Hayashi H, Tanabe H. Amyotrophic lateral sclerosis with hypertensive attacks: blood pressure changes in response to drug administration. Clin Auton Res 1996; 6: 241-244

3. Pavlovic S, Stevic Z, Milovanovic B, Milicic B, Rakocevic-Stojanovic V, Lavrnic D, Apostolski S. Impairment of cardiac autonomic control in patients with amyotrophic lateral sclerosis. Amyotroph Lateral Scler 2010; 11: 272-276

4. Shimizu T, Hayashi H, Kato S, Hayashi M, Tanabe H, Oda M. Circulatory collapse and sudden death in respirator-dependent amyotrophic lateral sclerosis. J Neurol Sci 1994; 124: 45-55

5. Blackman JA, Patrick PD, Buck ML, Rust RS Jr. Paroxysmal autonomic instability with dystonia after brain injury. Arch Neurol 2004; 61: 321-328

6. Ohno T, Shimizu T, Kato S, Hayashi H, Hirai S. Effect of tamsulosin hydrochloride on sympathetic hyperactivity in amyotrophic lateral sclerosis. Auton Neurosci 2001; 88: 94-98

7. Kitajima T, Kanbayashi T, Saito Y, Takahashi Y, Ogawa Y, Sugiyama T, Kaneko Y, Aizawa R, Shimizu T. Diazepam reduces both arterial blood pressure and muscle sympathetic nerve activity in human. Neurosci Lett 2004; 355: 77-80

8. Marty J, Gauzit R, Lefevre P, Couderc E, Farinotti R, Henzel C, Desmonts JM. Effects of diazepam and midazolam on baroreflex control of heart rate and on sympathetic activity in humans. Anesth Analg 1986; 65: 113-119 